\title{
Reward magnitude and a comment
}

\author{
GARVIN McCAIN, RICHARD WARD, and MICHAEL LOBB \\ University of Texas, Arlington, Texas 76109
}

\begin{abstract}
Marrero, Davis, and Seago (1973) have questioned the findings by McCain and others that performance differences between large- and small-reward groups disappear after extended training. Comments are made regarding their criticisms and experiment. Two studies in which a small-reward group ran slower early in acquisition and slightly faster than a large-reward group after extended training are also presented.
\end{abstract}

This is another in a series of papers (McCain, 1970, 1971; McCain, Dyleski, \& McElvain, 1971) questioning traditionally accepted assumptions regarding the effects of reward magnitude. These nine studies indicate that early in acquisition, groups given large reward run faster than small-reward groups, but these differences disappear after extended training. We are aware of at least 30 studies in which there were no asymptotic differences in large- and small-reward groups.

Recently, Marrero, Davis, and Seago (1973), sponsored in this journal by Robert Prytula, have questioned these results. They suggested three bases for their doubts. First, they raise a question as to whether the large-reward groups may be or may not be more satiated since studies from this laboratory typically have employed multiple trials (usually five) each day. Second, they cite a comment by Traupmann and Wong (1971) that a transformation of the data from one of our nine studies might indicate a difference existed late in training. An analysis of transformed individual scores for that particular study (McCain, 1971) revealed no significant terminal acquisition differences. We have several studies, including McCain et al. (1971, Experiment 1) and the present two, where small-reward groups run faster than the large-reward groups late in training. How transformation could reverse these differences is not clear. Third, Marrero et al. (1973) conclude that their study is contrary to the McCain findings.

While there is no question as to the propriety of Marrero et al. raising doubts about prior research, their study is a bit puzzling. First, they used a total of 50 acquisition trials. McCain et al. (1971) mention 50+ trials; McCain (1970) mentions 60 trials as the time when small-reward groups began to run as fast as largereward groups. In practice we have seen the group differences disappear anywhere from approximately 10 trials to about 100 trials. If previously published

Requests for reprints should be sent to Garvin McCain, Department of Psychology, University of Texas, Arlington, Texas 76019. This research was supported by a grant from the Organized Research Fund of the University of Texas at Arlington. The raw data, from this and any other studies from this laboratory, are available for the cost of reproduction. statements have led to confusion, it is regrettable. The present authors are not committed to any specific "magic" number of trials. Given the variations in animals, experimenters, equipment, etc., it would be foolhardy to do other than give rough approximations. Even if we are nailed to the $50+$ statement, which was intended simply as an empirical summary of those studies, Marrero et al.'s termination of training at exactly 50 trials hardly seems an adequate test.

Second, Marrero et al. (1973) reported their analyses of "... Trial Blocks 19-25 (the point in training at which asymptotic performance appeared to have been reached)." Inspection of their Figure 1 does not convince the present authors that asymptote has been reached. Happily, psychologists have statistical techniques that would allow "appeared" to be replaced by a clear and definite statement.

Third, it is unfortunate that Marrero et al. did not cite several pertinent studies including Black (1969) and Campbell, Batsche, and Batsche (1972). In a series of eight studies, Black found effects compatible with McCain et al. (1971). Campbell et al., in a well-designed study, used one trial per day, gave 120 trials, and stated, "Neither the confounding of drive level nor the use of single-pellet rewards are necessary to observe the reward magnitude collapse reported by Black (1969) and McCain et al. (1971)." The possible problem of satiation was obvious from the beginning of our reward magnitude research; it was very helpful to have a colleague in another laboratory examine the possible effects of satiation.

Fourth, two of Marrero et al.'s groups were anosmic rats. The small-reward group was significantly faster in the start section $(\mathrm{p}<.05)$ than the large-reward group. They explain this as follows: "The data reported here would suggest that anosmia, in addition to eliminating reward magnitude effects ....." "Elimination" seems to be an assumption after the fact that neatly disposes of an uncomfortable and apparently unexpected result. Incidentally, Marrero et al. show their analyses of variance (e.g., olfactory state by reward magnitude interaction) as having 1 and $19 \mathrm{df}$. Since they had a total of 20 subjects distributed in four groups, their use of $\mathrm{df}$ does seem somewhat unique. 
Finally, the Marrero et al. citation of McCain (1969) as one of the reward magnitude studies is puzzling. Groups received either one 1000-mg or $2245-\mathrm{mg}$ Noyes pellets and 40 trials before the rewards were reversed. The results were similar to the Crespi effect. Just how that study is of substantial concern in the present context is far from clear.

Since Marrero et al. have raised a question about transformed data, the present results will be presented as transformations $(1 / \log \bar{x}+1)$ in Experiment $I$ and $\log [(1 / x)+1]$ in Experiment II.

Experiment I was one of six studies examining possible interactions between drive level and reward magnitude. In each case, the magnitude results were compatible with those presented here. Due to lack of space, drive data will be presented in another paper. Briefly, the results indicated no drive level by reward magnitude interactions. Experiment II was a reward magnitude shift study. The data presented are from the control groups.

\section{EXPERIMENT I}

\section{Method $^{1}$}

Subjects. The subjects were 2490 -day-old naive rats of the Holtzman strain.

Apparatus. A plywood straight alley $160 \mathrm{~cm}$ long was used. Run speed (RS) was measured over a $45-\mathrm{cm}$ section of the alley and goal speed (GS) over $30.5 \mathrm{~cm}$.

Procedure. The subjects were divided into four groups, with large (LR) and small reward (SR) and high (HD) and low drive (LD) as the conditions. LR was $28 \mathrm{sec}$ access to wet mash, SR was $7 \mathrm{sec}$ access. HD was reduction to $80 \%$ initial body weight, LD was reduction to $90 \%$. Subjects explored the apparatus on 2 successive days for approximately $5 \mathrm{~min} /$ day. Beginning on the third day, five acquisition trials per day were given for 10 days. This was followed by 10 extinction trials. Since the extinction data do not bear directly on the present problem, they are not presented but are available on request. Briefly, the extinction results of Experiment I support McCain et al. (1971), while Experiment II does not.

\section{Results}

Comparison of reward magnitudes in the early part of acquisition (Trials 2-15) reveals no significant differences in RS $(F<1)$ but there is a significant difference in the GS $(F=4.50, \mathrm{df}=1 / 20, \mathrm{p}<.05)$. In both measures LR ran faster.

As may be seen in Figure 1, during terminal acquisition $\mathrm{SR}$ is faster than $\mathrm{LR}$ in the run section, but the difference was not significant $(F=2.37$, df $=$ $1 / 20, p>.10)$. There was a significant Reward by Trials interaction in $\mathrm{GS}(\mathrm{F}=2.78, \mathrm{df}=4 / 80, \mathrm{p}<.05)$. A t test between the first block of GS trials shown in Figure 2 was not significant $(\mathrm{t}<1)$.

Since there was some concern as to a possible satiation effect, comparisons were made between first and last trials of each day for the first 3 and last 3 days of acquisition. The only significant differences on the

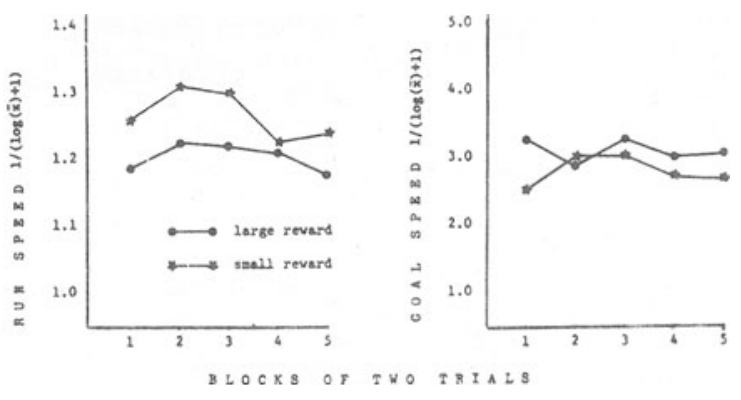

Figure 1. Terminal acquisition run and goal speeds: Experiment I.

direct difference $t$ tests was for SR on Day $10(t=2.33$, $\mathrm{df}=11, \mathrm{p}<.05)$, where they slowed down in GS. However, there is a significant difference for SR in RS for Day 9 , where they speeded up over trials $(t=2.42$, $\mathrm{df}=11, \mathrm{p}<.05)$.

\section{EXPERIMENT II}

\section{Method}

Subjects and Apparatus. The subjects were 22 naive 110-dayold male rats of the Holtzman strain. Two subjects became ill and were dropped. The apparatus was the same as in Experiment I.

Procedure. Throughiout the experiment, subjects were on a daily ration of $14 \mathrm{~g}$ of lab chow; food in the alley was subtracted from cage feeding. Following the seventh day of deprivation, each subject explored the alley for $5 \mathrm{~min}$ daily. The exploration procedure involved the operation of the switches, doors, photobeams, and timers which would be a part of the experimental sessions. The apparatus was regularly cleaned with Pine Sol during the exploration periods. Food dishes containing $45-\mathrm{mg}$ Noyes pellets were placed in the home cages during this period. The subjects were weighed, and matched groups were formed on this basis. Running orders were systematically varied across the squads of six subjects. Following the 10th day of deprivation, the experimental sessions began. Group SR received one $45-\mathrm{mg}$ Noyes pellet, Group LR received $1145-\mathrm{mg}$ pellets on each trial. On the first day of training, two trials were given, three trials on the second day, and five trials on each succeeding day, for a total of 170 trials. Subjects were removed from the goalbox immediately following consumption of the available food.

\section{Results}

The results are rather simple and straightforward, as can be seen in Figure 2. Group LR initially (Days $1-10)$ ran faster $(\mathrm{F}=5.00, \mathrm{df}=1 / 18, \mathrm{p}<.05)$. Over the latter stages of training (Days 25-35), SR ran faster but the difference was not significant $(F<1)$. GS was compatible with RS except that initial differences were smaller and the terminal differences were comparably larger.

We compared first and last trials for each group over each of the last 10 days of acquisition for GS and RS. Of the 40 direct difference $t$ tests, not a single one was significant. SR showed a somewhat greater tendency to slow down over trials than did LR. 


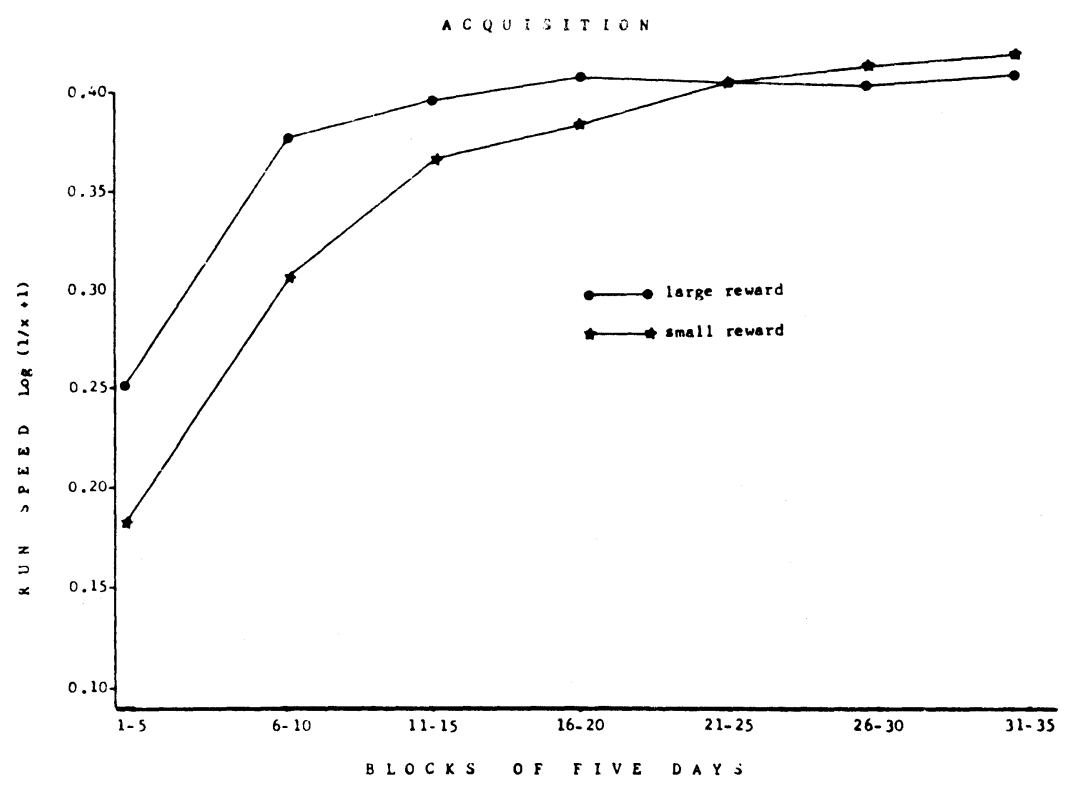

Figure 2. Acquisition run speed: Experiment II. Note that the crossover takes place at about 100 trials.

\section{DISCUSSION}

These experiments, along with numerous others, do not fit traditional assumptions about the effects of reward magnitude. Our large-reward groups run faster early in training, but late in training the differences disappear and either large- or smallreward groups may run slightly faster.

The first and last trial comparisons certainly give no indication of any significant or substantial differential slowdown for the large- and small-reward groups. Of 60 direct differences $t$ tests, only 2 were significant; those were in the same group but in opposite directions. We had performed similar analyses on previous data but did not report them, since the results of Campbell et al. (1972) seemed an adequate answer to the satiation question.

Earlier (McCain, 1970) we ran large- and small-reward partial reinforcement groups. Early in training the large-reward group ran faster; late in training there were no differences. Capaldi and Freese (1974) have obtained similar results. More recently, Meyers and Anderson (1975), utilizing barpresses and 1 and 15 45-mg Noyes pellets, found no terminal acquisition differences after 90 or 300 trials.

It is interesting to note that data with similar implications have been reported from a human learning paradigm. Cox, Weitz, and Lieberman (1975), sponsored in this journal by Judson S. Brown, found no performance differences between groups of human subjects given large or small reward (number of points toward course grade).

\section{REFERENCES}

Bl.ACK, R. W. Incentive motivation and the parameters of reward in instrumental conditioning. In W. J. Arnold and D. Levine (Ed.), Nebraska symposium on motivation. Lincoln: University of Nebraska Press, 1969.

Campbell, P. E., Batsche, C. J., \& Batsche, G. M. Spaced trials reward magnitude effects in the rat: Single versus multiple food pellets. Journal of Comparative and Physiological Prychology, 1972, 81, 360-364.
Capaldi, E. J., \& Freese, M. R. Partial reward training level and reward magnitude: Effects on acquisition and extinction. Learning and Motivation, 1974, 5. 299-310.

Cox, W. M., Weitz, J. R., \& Lieberman, L. R. Shifts in magnitude of reward with humans in the "straightway." Bulletin of the Psychonomic Society, 1975, 6, 1-3.

LaWrence, S. M., \& Anderson, G. J. Acquisition and extinction following extended partial reinforcement training under small or large reward. Bulletin of the Psychonomic Society, 1975, 6, 198-200.

Marrero, B., Davis, S. E., \& Seago, J. D. Runway performance of normal and anosmic rats as a function of reward magnitude: A preliminary report. Bulletin of the Psychonomic Society, 1973, 2, 237-376.

MCCAIN, G. Different levels of performance with equivalent weights of reward. Psychonomic Science, 1969, 14, 2-3.

McCaIN, G. Reward magnitude and instrumental responses: Consistent and partial reward. Psychonomic Science. 1970, 19, 139-141.

McCaIn, G. Traupmann and Wong: A reply. Psychonomic Science, 1971, 23, 14-15.

McCain, G., Dyleski, K., \& McElvain, G. Reward magnitude and instrumental responses: Consistent reward. Psychonomic Monograph Supplements, 1971, 3(16, Whole No. 48).

Meyers, L. S., \& Anderson, G. J. Acquisition and extinction following extended partial reinforcement training under small or large reward. Bulletin of the Psychonomic Society, 1975, 6. 198-200.

Traupmann, K. L., \& Wong, P. T. P. Reward magnitude and instrumental responses: A comment. Psychonomic Science, 1971, 23, 13-14.

\section{NOTE}

1. Due to the amount of material covered, the apparatus and procedures are given in brief (details are available).

(Received for publication September 8, 1975.) 